\title{
A RANK PRESERVING FLOW ALGORITHM FOR QUADRATIC OPTIMIZATION PROBLEMS SUBJECT TO QUADRATIC EQUALITY CONSTRAINTS
}

\author{
John B. Moore \\ Department of Systems Engineering \\ Research School of Information Sciences and Engineering \\ Australian National University \\ ACT 0200, Australia
}

\author{
Danchi Jiang* \\ Department of Mechanical and Automation Engineering \\ Chinese University of Hong Kong \\ Shatin, N. T. Hong Kong \\ Email: dcjiang@mae.cuhk.edu.hk
}

\begin{abstract}
This paper concerns quadratic programming problems subject to quadratic equality constraints such as arise in broadband antenna array signal processing and elsewhere. At first, such a problem is converted into a semidefinite programming problem with a rank constraint. Then, a rank preserving flow is used to accommodate the rank constraint. The associated gradient formulas are carefully developed. The convergence of the resulted algorithm is also guaranteed. Our approach is demonstrated by a numerical experiment.
\end{abstract}

\section{PROBLEM DESCRIPTION}

Consider the following general quadratic programming problem:

$$
\begin{array}{cl}
\min & J_{0}(X):=\operatorname{tr}\left(X^{\top} Q_{0} X+B_{0} X\right) \\
\text { subject to: } & J_{i}(X):=\operatorname{tr}\left(X^{\top} Q_{i} X+B_{i} X\right)=c_{i}, \\
& i=1,2, \ldots, m .
\end{array}
$$

where $X \in \Re^{p \times q}, Q_{0}$ is a positive definite matrix, and $Q_{i}, i=1,2, \ldots, m$ are positive semi-definite matrices. A linear constraint is covered as a special case where the matrix $Q_{i}$ for the corresponding index $i$ is a zero matrix.

For given generic $Q_{i}, i=1,2, \ldots, m$, it is a difficult task to solve the problem (1) (2). One of the main reasons is that the admissible set in the generic case is disconnected. Hence, any gradient based methods for the searching of the optimal solution is bound to lead to a local optimal. Another reason is that, eventhough one can use a gradient based method to solve it, the computation of the gradient of the cost function is complicated for problems of large size. Also

\footnotetext{
* Partly supported by the funding of the activities of the Cooperative Research Centre for Robust and Adaptive Systems by the Australian Government under the Cooperative Research Centres Program.
}

because this problem is not convex, quadratic equality constraints can not be touched by the popular interiorpoint polynomial methods such as reported in [1], [3] and [4].

However, this quadratic optimization problem arises in applications. For instance, in the area of broad band antenna array signal processing, the global minimum of such a problem leads to an optimal design. See [2] for details. It is of interest to developed appropriate algorithm to solve this problem.

In this paper, motivated by a desire to achieve improved optimization techniques, we convert the quadratic programming problem into an optimization problem of a linear matrix function subject to linear equality, linear matrix inequality, and matrix rank constraints. Then, an algorithm is developed to solve the converted problem based on gradient flow with respect to certain Riemannian metric.

The paper is divided into four sections. Section 1 introduces the problem of interest. Section 2 converts the problem into an optimization problem for linear matrix function subject to linear equality, linear matrix inequality, and matrix rank constraints. The related convex problem is also discussed. Sections 3 is devoted to the development of an algorithm. The variable evolves in a set of positive semi-definite matrices of a fixed rank. Section 4 contains a numerical experiment using the algorithm developed.

\section{CONVERTED PROBLEMS}

First, we note the following lemma.

Lemma 1 The matrix equality

$$
Y=X X^{\top}
$$

is equivalent to

$Z:=\left(\begin{array}{cc}Y & X \\ X^{\top} & I_{q}\end{array}\right) \geq 0, \quad \operatorname{rank}\left(\begin{array}{cc}Y & X \\ X^{\top} & I_{q}\end{array}\right)=q$ 
We also note that the original problem is equivalent to the problem defined as follows:

$$
\begin{aligned}
\min & J_{0}=\operatorname{tr}\left(Q_{0} Y+B_{0} X\right) \\
\text { subject to: } & \left\{\begin{array}{l}
J_{i}=\operatorname{tr}\left(Q_{i} Y+B_{i} X\right), \\
i=1,2, \ldots, m . \\
Y=X X^{\top} \text { i.e. (3) holds. }
\end{array}\right.
\end{aligned}
$$

The original problem is equivalent to the following

$$
\min \operatorname{tr}\left(K_{0} Z\right)
$$

subject to: $\quad\left\{\begin{array}{l}\operatorname{tr}\left(K_{i} Z\right)=c_{i}, \quad i=1,2, \ldots, m . \\ Z^{\top}=Z, \quad Z \geq 0, \quad \operatorname{rank}(Z)=q,(8) \\ \operatorname{diag}\left(0, I_{q}\right) Z \operatorname{diag}\left(0, I_{q}\right)=\operatorname{diag}\left(0, I_{q}\right)\end{array}\right.$

where

$$
K_{i}=\left(\begin{array}{cc}
Q_{i} & \frac{1}{2} B_{i}^{\top} \\
\frac{1}{2} B_{i} & 0
\end{array}\right), \quad i=0,1, \ldots, m .
$$

To simplify the formulation of (8), note that the last equality constraint can be converted into a group of equality constraints on the trace of some linear matrix function. More specifically, it is equivalent to the following $\frac{q(q+1)}{2}$ equality constraints:

$$
\begin{array}{r}
\operatorname{tr}\left(e_{j}^{\top} e_{j} Z\right)=1, \quad j=p+1, p+2, \ldots, p+q . \\
\operatorname{tr}\left(\left[e_{u}+e_{v}\right]^{\top}\left[e_{u}+e_{v}\right] Z\right)=2, \\
p+1 \leq u, v \leq p+q, \quad u \neq v
\end{array}
$$

where $e_{j}$ is the the $j$-th elementary column vector whose $j$-th component is 1 and other components are zero. Let $c_{i}=1$ for $m+1 \leq i \leq m+q, c_{i}=2$ for $m+q+1 \leq i \leq L$, where $L:=m+\frac{q(q+1)}{2}$ and let the $K_{i}$ be the corresponding coefficient matrices in the equalities (10) and (11). The optimization problem defined by (7) (8) now is converted into the following form:

$$
\min \operatorname{tr}\left(K_{0} Z\right)
$$

subject to:

$$
\left\{\begin{array}{l}
\operatorname{tr}\left(K_{i} Z\right)=c_{i}, \quad i=1,2, \ldots, L \\
Z^{\top}=Z, \quad Z \geq 0
\end{array}\right.
$$

and:

$$
\operatorname{rank}(Z)=q \text {. }
$$

This problem is easy to solve if the rank condition is removed. In fact, the optimal problem defined by (12) and (13) is a standard semi-definite programming problem. As an important class of convex problem, it has been extensively studied recently and is known to be solved by interior-point methods in polynomial time with respect to the size of the problem. For details, see [1]. It is also referred to as a linear matrix inequality (LMI).

The following results concern properties of the corresponding solution.
Theorem 1 Suppose $X^{\star}$ is the optimal solution to the problem (12) (13).

(1). If $X^{\star}$ is of full rank, then, $K_{0}$ can be represented as a linear combination of $K_{i}, \quad i=1,2, \ldots, L$. i.e., there exist real numbers $k_{i}, \quad i=1,2, \ldots, L$ such that $K_{0}=\sum_{i=1}^{L} k_{i} K_{i}$. In this case, the cost (12) is invariant for all admissible solutions.

(2). If $X^{\star}$ is not of full rank, let $N_{j}, \quad j=1, \ldots, r$ for integer $r=p+q-\operatorname{rank}\left(X^{\star}\right)$ be independent null vectors of it. Then, $K_{0}$ is a linear combination of $K_{i}, \quad i=1,2, \ldots, L$ and $N_{i} N_{i}^{\top}, \quad i=1, \ldots, r$. In this case, all positive semi-definite matrices $Z$ that satisfy the following conditions

$$
\begin{aligned}
\operatorname{tr}\left(K_{i} Z\right) & =c_{i}, \quad i=1,2, \ldots, L \\
\operatorname{tr}\left(N_{i} N_{i}^{\top} Z\right) & =0, \quad i=1, \ldots, r .
\end{aligned}
$$

are the optimal solutions and have the same cost.

(3). If the optimal solution for the problem (12) (13) is not unique, there are a group of common zero eigenvectors $N_{i}^{\star}, i=1,2, \ldots, s$ for some positive integer $s$ such that (a) $K_{i}, i=1,2, \ldots, m$ and $N_{i}^{\star}, i=$ $1,2, \ldots, s$ are independent and (b) $K_{0}$ is a linear combination of $K_{i}, i=1,2, \ldots, m$ and $N_{i}^{\star}, i=1,2, \ldots, s$.

Proof (1). Since $X^{\star}$ is of full rank, there exists a neighborhood of it where all symmetric matrices are positive definite. Furthermore, there exists a positive real number $\delta>0$, such that, for all symmetric matrix $H$ satisfying

$$
\begin{array}{r}
\|H\|_{F}=\sqrt{H H^{\prime}} \leq \delta, \\
\operatorname{tr}\left(K_{i} H\right)=0, i=1,2, \ldots, L,
\end{array}
$$

$\left(X^{\star}+H\right)$ is an admissible solution for the constraints (13) and $\operatorname{tr}\left(K_{0}\left(X^{\star}+H\right)\right) \geq \operatorname{tr}\left(K_{0} X^{\star}\right)$. This leads to $\operatorname{tr}\left(K_{0} H\right)=0$. Therefore, $K_{0}$ is a linear combination of $K_{i}, i=1,2, \ldots, L$. Obviously, the cost of any admissible point is also the corresponding linear combination of $c_{i}$, and hence a constant.

(2). Let $N^{\perp}$ be defined as:

$$
\begin{aligned}
N^{\perp}=\quad & \left\{Y \in \Re^{(p+q) \times(p+q)}:\right. \\
& \left.Y^{\top}=Y, Y N_{i}=0, i=1, \ldots, r .\right\} .
\end{aligned}
$$

Then, it is straight forward to show that there is a positive real number $\tilde{\delta}>0$ such that, for all $H \in N^{\perp}$, if $\|H\|_{F} \leq \tilde{\delta}$, then, $X^{\star}+H$ is positive semi-definite. Following the argument for (1) we obtain the validity of (2).

(3). First, we claim that for two optimal solutions $X_{1}$ and $X_{2}, \frac{1}{2}\left(X_{1}+X_{2}\right)$ is also an optimal solution and, furthermore, only a common zero eigenvector of $X_{1}$ and $X_{2}$ can be its zero eigenvector. 
The first part of the claim is clearly implied by the convexity of the problem and its constraints. For the second part, assume $V$ is a zero eigenvector of $\frac{1}{2}\left(X_{1}+\right.$ $\left.X_{2}\right)$. Then, $V^{\top}\left(X_{1}+X_{2}\right) V=0$. Since both $X_{1}$ and $X_{2}$ are positive semi-definite, $V^{\top} X_{1} V=0$ and $V^{\top} X_{2} V=$ 0 . Therefore, $V$ is a common zero eigenvector of $X_{1}$ and $X_{2}$.

Hence, if the property of (3) does not hold, there exists a group of optimal solutions $Z_{i}, i=1,2, \ldots, h$ for some positive integer $h$ such that their common eigenvectors, denoted as $U_{j}, j=1, \ldots, g$, together with $K_{i}, i=1,2, \ldots, m$ can not be organized as a linear representation for $K_{0}$. Similarly, we can show that $\frac{1}{h}\left(Z_{1}+Z_{2}+\ldots+Z_{h}\right)$ is an optimal solution and $U_{j}, j=1, \ldots, g$ are a group of its zero eigenvectors. This is a contradiction to the results in (2). Hence the proof is complete.

It is possible that the optimal solution to the convex programming problem defined by (12) and (13) has $p$ zero eigenvectors. If it is the case, based on Theorem 1 , the optimal solution of the convex problem and that of the optimization problem defined by (12), (13) and (14) coincide. However, numerical results conducted by the authors imply that it is not always the case. In the next two sections, an algorithm will be developed to search for a solution to the problem subject to a rank constraint.

\section{RANK PRESERVING FLOW ALGO- RITHM}

Let the set $P(q)$ denote all $(p+q)$-dimensional positive semi-definite matrices of rank $q$. From Proposition 1.1 in [5, page 134], we know that $P(q)$ is a connected smooth manifold and its tangent space is calculated as:

$$
T_{Z} P(q)=\left\{\Delta Z+Z \Delta^{\top} \mid \Delta \in \Re^{(p+q) \times(p+q)}\right\} .
$$

First of all, let us show that the cost function (12) has compact sublevel sets. Since $K_{0}$ is in the form of $\left(\begin{array}{cc}Q_{0} & \frac{1}{2} B_{0}^{\top} \\ \frac{1}{2} B_{0} & 0\end{array}\right)$, one can always choose a positive definite matrix $C$ of an appropriate dimension such that $\bar{K}:=\left(\begin{array}{cc}Q_{0} & \frac{1}{2} B_{0}^{\top} \\ \frac{1}{2} B_{0} & C\end{array}\right)$ is positive definite. Therefore, $\operatorname{tr}\left(K_{0} Z\right)=\operatorname{tr}(\bar{K} Z)-\operatorname{tr}(C)$, bearing in mind that $Z$ is in the form of $\left(\begin{array}{cc}Y & X \\ X^{\top} & I_{q}\end{array}\right)$. Hence, the fact that $\operatorname{tr}\left(K_{0} Z\right)$ is bounded implies that $Z$ is bounded.

Based on the compactness of sublevel sets of the cost function $J_{0}$ defined by (12), the following two properties concerning the optimal point set hold:
- The set of all optimal point contains at most finite number of connected closed branches. These branches are isolated.

- Any algorithm, as long as it guarantees the descent of the cost function $J_{0}$, will converge to one of those connected branches.

Now we are going to compute the gradient of the cost function. At any point $Z \in P(q)$, decompose $\Re^{(p+q) \times(p+q)}$ as $S \oplus S^{\perp}$ such that $S$ is the kernel of the linear map:

$$
\begin{aligned}
\pi: \Re^{(p+q) \times(p+q)} & \mapsto \Re^{(p+q) \times(p+q)}, \\
\pi(\Delta) & =\Delta Z+Z \Delta^{\top} .
\end{aligned}
$$

Denote $\operatorname{Pr}$ as the corresponding projection such that $\operatorname{Pr}(S)=0$. Define a Riemannian metric as:

$$
\ll \Delta_{1}, \Delta_{2} \gg:=2 \operatorname{tr}\left\{\left[\operatorname{Pr}\left(\Delta_{1}\right)\right]^{\top} \operatorname{Pr}\left(\Delta_{2}\right)\right\} .
$$

Since

$\left.D J_{0}\right|_{Z}(\Delta)=\operatorname{tr}\left\{K_{0}\left(\Delta Z+Z \Delta^{\top}\right)\right\}=2 \operatorname{tr}\left(Z K_{0} \Delta\right)$,

the gradient of $J_{0}$ associated with the Riemannian metric defined by (16) is calculated as:

$$
\operatorname{grad} J_{0}=K_{0} Z^{2}+Z^{2} K_{0} .
$$

The projected gradient onto the constrained surface by (13) can be calculated as:

$$
\begin{aligned}
\operatorname{Grad} J_{0}= & \left(K_{0}-\sum_{i=1}^{L} k_{i} K_{i}\right) Z^{2}+ \\
& Z^{2}\left(K_{0}-\sum_{i=1}^{L} k_{i} K_{i}\right)
\end{aligned}
$$

where $k_{i}$ satisfy:

$$
\begin{aligned}
& \sum_{i=1}^{L} k_{i} \operatorname{tr}\left\{\left[\operatorname{Pr}\left(K_{i} Z\right)\right]^{\top} \operatorname{Pr}\left(K_{j} Z\right)\right\}= \\
& \operatorname{tr}\left\{\left[\operatorname{Pr}\left(K_{0} Z\right)\right]^{\top} \operatorname{Pr}\left(K_{j} Z\right)\right\}, \quad j=1,2, \ldots, L .
\end{aligned}
$$

The associated negative gradient flow is defined as:

$$
\dot{Z}=-\operatorname{Grad} J_{0} \text {. }
$$

Along any trajectory of this flow, the cost function $J_{0}$ always decreases until arriving an equilibrium point. 


\section{NUMERICAL SIMULATION}

In this section, we conduct a numerical experiment using the gradients developed in Sections 3. The parameters for the problem defined by (1) and (2) are chosen by random number generator randn in Matlab as:

$$
\begin{array}{r}
Q 0=\left(\begin{array}{ccc}
0.3180 & 1.6065 & -0.9235 \\
-0.5112 & 0.8476 & -0.0705 \\
-0.0020 & 0.2681 & 0.1479
\end{array}\right), \\
Q 1=\left(\begin{array}{ccc}
1.5578 & 1.1226 & 0.4142 \\
-2.4443 & 0.5817 & -0.9778 \\
-1.0982 & -0.2714 & -1.0215
\end{array}\right), \\
Q 2=\left(\begin{array}{ccc}
-0.5077 & -0.7262 & -0.2091 \\
0.8853 & -0.4450 & 0.5621 \\
-0.2481 & -0.6129 & -1.0639
\end{array}\right), \\
B 0=\left(\begin{array}{c}
-0.5571 \\
-0.3367 \\
0.4152
\end{array}\right) ; \quad B 1=\left(\begin{array}{c}
0.3177 \\
1.5161 \\
0.7494
\end{array}\right), \quad C_{1}=0 ; \\
B 2=\left(\begin{array}{c}
0.3516 \\
1.1330 \\
0.1500
\end{array}\right), \quad C_{2}=0,
\end{array}
$$

for $X \in \Re^{3}, m=2$. The parameter matrices $K_{0}, K_{1}, K_{2}$ is calculated based on these parameters.

For the Rank Preserving Flow, we compute the Projection operator first.

$\operatorname{vec}(\pi \Delta)=\operatorname{Avec}(\Delta):=\left(Z^{\top} \otimes I+(I \otimes Z) P(4,4)\right) \operatorname{vec}(\Delta)$, where $\operatorname{vec}(\Delta)$ is the column vector where the column vectors of $\Delta$ are stacked in order, $\otimes$ is the matrix Kronecker product, and $P(4,4)$ is the fourth-order permutation matrices defined as:

$$
P(4,4):=\sum_{i=1}^{4} \sum_{j=1}^{4} E_{i, j} \otimes E_{i, j}^{\top},
$$

and $E_{i, j}$ is the matrix that the $(i, j)$-th component is 1 and the component elsewhere is zero. Therefore,

$$
\operatorname{vec}(\operatorname{Pr}(S))=\mathcal{A}^{+} \text {Avec }(S),
$$

where $\mathcal{A}^{+}$is the pseudo-inverse of the matrix $\mathcal{A}$.

Then, use ODE23 in Matlab to search for an optimal solution. The initial condition is chosen as

$$
Z 0=\left(\begin{array}{llll}
0 & 0 & 0 & 0 \\
\mathbf{0} & 0 & 0 & 0 \\
0 & 0 & 0 & 0 \\
0 & 0 & 0 & 1
\end{array}\right) .
$$

In Figure 1, we can see that, eventhough the cost function is not convex, the algorithm still converge quickly. The solution obtained is $X=$ $(0.0286,-0.0305,-0.2124)^{\top}$. The corresponding cost is $J_{0}=-0.0888$.

Another possible approach is to penalize the rank of the symmetric matrix $Z$ in the semidefinite programming problem by some means so as to obtain the minimal rank one. This approach may result in a convex problem. Further detail is to be investigated.

\section{References}

[1] Y. Nesterov and A. Nemirovsky (1994), Interiorpoint polynomial methods in convex programming. Studies in Applied Mathematics, SIAM, 13.

[2] I. Thng, A. Cantoni and Y. H. Leung (1994), Analytical solutions to the optimization of a quadratic cost function subject to linear and quadratic equality constraints. to appear in Applied Mathematics and Optimizations.

[3] F. Jarre (1991), On the convergence of the method of analytic centers when applied to convex quadratic programs. Mathematical Programming 49, 341-358.

[4] S. Mehrotra and J. Sun (1991), A method of analytic centers for quadratically constrained convex quadratic programs. SIAM J. Numer. Anal. Vol. 28 , No. 2, pp. 529-544.

[5] U. Helmke and J. B. Moore (1994), Optimization and Dynamical Systems Springer-Verlag, London.

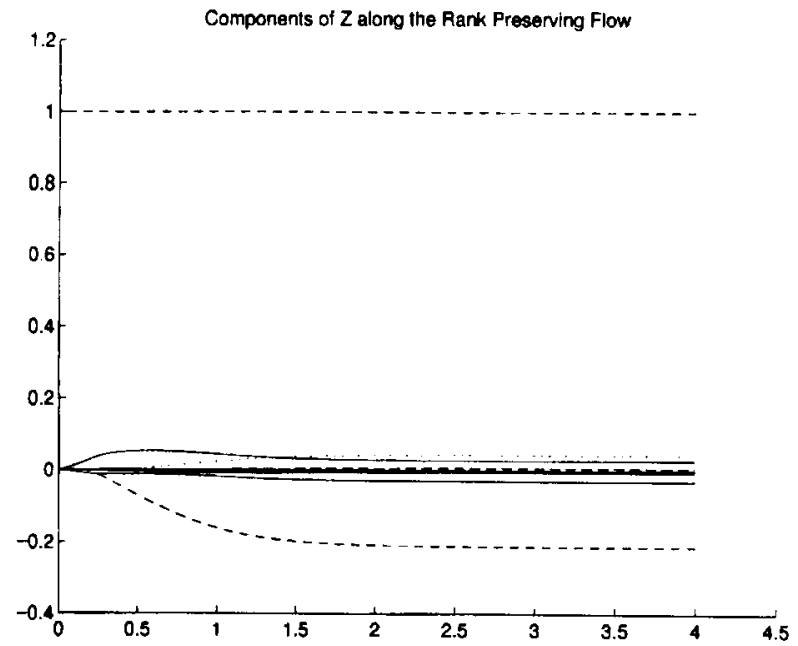

Figure 1. The convergence of all components of $Z$ along the Rank Preserving Flow.

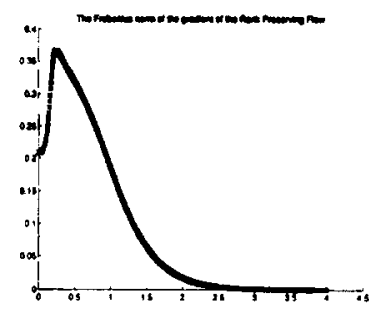

Figure 2. The Frobenius norm of the gradient along the Rank Preserving Flow

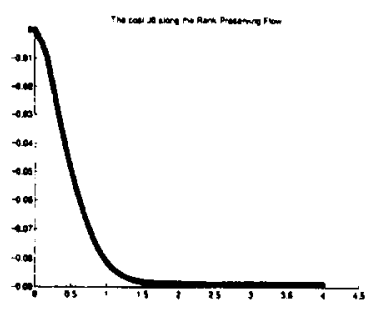

Figure 3. The cost $J_{0}$ along the Rank Preserving Flow 